\title{
Different types of bursting calcium oscillations in non-excitable cells
}

\author{
Matjaž Perc, Marko Marhl * \\ Department of Physics, Faculty of Education, University of Maribor, Koroška cesta 160, SI-2000 Maribor, Slovenia
}

Accepted 24 February 2003

\begin{abstract}
In the paper different types of bursting $\mathrm{Ca}^{2+}$ oscillations are presented. We analyse bursting behaviour in four recent mathematical models for $\mathrm{Ca}^{2+}$ oscillations in non-excitable cells. Separately, regular, quasi-periodic, and chaotic bursting $\mathrm{Ca}^{2+}$ oscillations are classified into several subtypes. The classification is based on the dynamics of separated fast and slow subsystems, the so-called fast-slow burster analysis. For regular bursting $\mathrm{Ca}^{2+}$ oscillations two types of bursting are specified: Point-Point and Point-Cycle bursting. In particular, the slow passage effect, important for the Hopf-Hopf and SubHopf-SubHopf bursting subtypes, is explained by local divergence calculated for the fast subsystem. Quasi-periodic bursting $\mathrm{Ca}^{2+}$ oscillations can be found in only one of the four studied mathematical models and appear via a homoclinic bifurcation with a homoclinic torus structure. For chaotic bursting $\mathrm{Ca}^{2+}$ oscillations, we found that bursting patterns resulting from the period doubling root to chaos considerably differ from those appearing via intermittency and have to be treated separately. The analysis and classification of different types of bursting $\mathrm{Ca}^{2+}$ oscillations provides better insight into mechanisms of complex intra- and intercellular $\mathrm{Ca}^{2+}$ signalling. This improves our understanding of several important biological phenomena in cellular signalling like complex frequency-amplitude signal encoding and synchronisation of intercellular signal transduction between coupled cells in tissue.
\end{abstract}

(c) 2003 Elsevier Science Ltd. All rights reserved.

\section{Introduction}

Bursting refers to the phenomenon in which a variable of a dynamical system alternates between a silent and an active phase. During the silent phase, the variable remains quiescent, whereas the active phase is characterized by rapid oscillations. During the last three decades, many experimental [1-9] as well as theoretical studies [10-19] of different bursting patterns have been published. Bursting was first reported for the electrical activity of the neuron $\mathrm{R}_{15}[1,2]$. Afterwards, bursting was found to be the primary mode of electrical behaviour in many other neuron types, like for example thalamic neurons [4-6], AB neurons [7] and dopaminergic neurons of the mammalian midbrain [9]. Bursting oscillations have also been observed in pancreatic $\beta$-cells $[3,8]$. It was found that the type of bursting plays a vital role in determining the excitability and hence neuro-computational properties of neurons $[17,19]$.

Recently, the role of bursting oscillations for intracellular $\mathrm{Ca}^{2+}$ signalling has become of interest. The importance of cytosolic calcium is well established in a large variety of cell types. In excitable as well as in non-excitable cells a significant part of signal transduction from receptors at the cell membrane to enzymes, controlling the complex behaviour of the biological systems, is performed by the oscillatory changing of free cytosolic calcium concentration. These oscillations regulate many cellular processes from egg fertilization to cell death [20]. The mechanisms of these oscillations have been intensely investigated both from experimental and theoretical point of view (for review see $[21,22]$ ).

${ }^{*}$ Corresponding author. Tel.: +386-2-2293643; fax: +386-2-2518180.

E-mail address: marko.marhl@uni-mb.si (M. Marhl). 
Bursting oscillations of free cytosolic calcium were found experimentally in excitable (cf. [23]) as well as in nonexcitable cells [24-27]. Trying to explain the mechanism of bursting $\mathrm{Ca}^{2+}$ oscillations, several mathematical models for complex $\mathrm{Ca}^{2+}$ oscillations in excitable [23] as well as in non-excitable cells [28-31] were proposed. Here, we focus on the models that describe bursting $\mathrm{Ca}^{2+}$ oscillations in non-excitable cells [28-31]. The first model, describing bursting $\mathrm{Ca}^{2+}$ oscillations in non-excitable cells, was proposed by Shen and Larter [28]. The functioning of the model bases on two main mechanisms, i.e. the calcium-induced calcium release (CICR) [32] and the inositol trisphosphate crosscoupling (ICC) $[33,34]$. There are three variables in the model: free $\mathrm{Ca}^{2+}$ concentration in the cytosol $\left(\mathrm{Ca}_{\text {cyt }}\right)$, free $\mathrm{Ca}^{2+}$ concentration in the endoplasmic reticulum (ER) $\left(C a_{\text {er }}\right)$, and the inositol trisphosphate concentration in the cytosol $\left(I P_{3}\right)$ (for equations see Model 1 in Appendix A). Another CICR based model with the same set of model variables as in the previous model [28] was proposed by Borghans et al. [29], however, with focusing on the effect of cytosolic calcium on the degradation of inositol trisphosphate (for equations see Model 2 in Appendix A). Another modelling approach represent models proposed by Marhl et al. [30] and Kummer et al. [31]. In the model by Marhl et al. [30], the important role of mitochondria [35] and cytosolic $\mathrm{Ca}^{2+}$ binding proteins [36,37] is taken into account. The three main variables that describe the dynamics of the model system are: free $\mathrm{Ca}^{2+}$ concentration in the cytosol $\left(\mathrm{Ca}_{\text {cyt }}\right)$, free $\mathrm{Ca}^{2+}$ concentration in the ER $\left(\mathrm{Ca}_{\mathrm{er}}\right)$, and the free $\mathrm{Ca}^{2+}$ concentration in the mitochondria $\left(\mathrm{Ca}_{m}\right)$ (for equations see Model 3 in Appendix A). The model proposed by Kummer et al. [31] incorporates the feedback inhibition on the initial agonist receptor complex by $\mathrm{Ca}^{2+}$ and activated phospholipase C (PLC), as well as receptor type-dependent self-enhanced behaviour of the activated $G_{\alpha}$ subunit. Consequently, the three main variables are: the concentration of the active $G_{\alpha}$ subunit $\left(G_{\alpha}\right)$, the concentration of the active PLC $(P L C)$ and the free $\mathrm{Ca}^{2+}$ concentration in the cytosol $\left(C a_{\text {cyt }}\right)$ (for equations see Model 4 in Appendix A).

Although the models for bursting $\mathrm{Ca}^{2+}$ oscillations in non-excitable cells were present for several years, different types of bursting $\mathrm{Ca}^{2+}$ oscillations appearing in these models were studied only moderately [29,38-41]. Several bursting patterns, including simple duplets, entangled regular as well as chaotic bursting $\mathrm{Ca}^{2+}$ oscillations were found. Additionally, the occurrence of birhythmicity [39] as well as trirhythmicity and intermittency for chaotic bursting $\mathrm{Ca}^{2+}$ oscillations [40] was examined. However, a mathematical classification of different types of bursting was made only for the model proposed by Kummer et al. [31,41]. In the present paper, we provide a comparative mathematical classification of bursting types for all four recently proposed mathematical models [28-31] that account for complex intracellular calcium oscillations in non-excitable cells. The models are not described in details, however, for each model the basic model frame with the complete set of model equations and parameter values is given in Appendix A. For the classification of bursting $\mathrm{Ca}^{2+}$ oscillations, we use the scheme proposed by Izhikevich [17]. First, in the following section methods for bursting analysis are presented. In Section 3 bursting $\mathrm{Ca}^{2+}$ oscillations are mathematically analysed and classified. The classification is carried out separately for regular, quasi-periodic, and chaotic bursting $\mathrm{Ca}^{2+}$ oscillations. The biological importance of our results is discussed in terms of information encoding, signal flexibility and coupling properties of the cells.

\section{Methods}

\subsection{Fast-Slow burster analysis}

The mathematical analysis of bursting $\mathrm{Ca}^{2+}$ oscillations was carried out by the method proposed by Rinzel [42]. By this method, the system is separated into a "fast" and a "slow" subsystem. Therefore, we address to it as the Fast-Slow burster analysis. Each variable that changes significantly over the duration of each bursting spike is a part of the "fast" subsystem whereas the variables that significantly change only over the duration of the whole bursting phase represent the "slow" subsystem. We determine the type of bursting by the bifurcation analysis of the "fast" subsystem where the "slow" variables are used as bifurcation parameters.

\subsection{Bifurcation analysis and numerical integration}

The bifurcation analysis and the numerical integration of model equations was carried out with the numerical continuation software AUTO97 [43] and XPPAUT [44].

\subsection{Analysis of quasi-periodicity and chaos}

Quasi-periodicity and chaotic behaviour are determined by Lyapunov exponents, which were calculated with the algorithm proposed by Wolf et al. [45]. The results about the type of oscillations were verified by the Fourier analysis. 
We examined the characteristics of quasi-periodic bursting $\mathrm{Ca}^{2+}$ oscillations in the phase space only; whereas for chaotic $\mathrm{Ca}^{2+}$ oscillations also the transition to chaos was studied by bifurcation diagrams and return maps.

\section{Results}

We analyse bursting $\mathrm{Ca}^{2+}$ oscillations for four mathematical models [28-31] in which these complex $\mathrm{Ca}^{2+}$ oscillations can be found. Separately, we treat regular, quasi-periodic and chaotic bursting $\mathrm{Ca}^{2+}$ oscillations. Regular bursting $\mathrm{Ca}^{2+}$ oscillations are classified according to the scheme proposed by Izhikevich [17] into two main categories: PointPoint and Point-Cycle bursting. Each of these categories can be further classified in dependence on two bifurcations, which influence the transition from silent to active phase and vice versa. For example, if the active phase of bursting oscillations starts with a fold bifurcation and ends with a supercritical Hopf bifurcation, this type of bursting oscillation is called Fold-Hopf bursting. Moreover, this type of bursting oscillation can be further specified as Fold-SubHopf bursting, for example, in case of a subcritical Hopf bifurcation. In many cases, in addition to the two bifurcations responsible for the onset and termination of the active bursting phase there are also other bifurcations present. Therefore, the classification of bursting oscillations can have further subtypes according to these additional bifurcations (for review see [17]). Besides regular bursting $\mathrm{Ca}^{2+}$ oscillations, we also study quasi-periodic and chaotic bursting $\mathrm{Ca}^{2+}$ oscillations. These non-regular bursters can have the same origin as regular bursters e.g.: Point-Point or Point-Cycle basis. However, in addition to this classification also different roots to chaos have to be considered, since they contribute to qualitatively different bursting patterns. For chaotic bursting $\mathrm{Ca}^{2+}$ oscillations, we analyse two types of transition to chaos, i.e. period doubling and intermittency.

\subsection{Regular periodic bursting}

\subsubsection{Point-Point bursting}

Bursting $\mathrm{Ca}^{2+}$ oscillations of the Point-Point type are very common in the models for cytosolic $\mathrm{Ca}^{2+}$ oscillations [28-30]. The characteristics of the Point-Point bursting can be demonstrated by the fast-slow burster analysis (see Section 2). As results of this analysis, the bifurcation diagrams of slow subsystems are shown together with trajectories of the complete system. The main characteristic of the Point-Point bursting is that the silent as well as the active phase of bursting is associated to a steady state in the fast subsystem. This means that the fast subsystem does not have a limit cycle attractor for any value of the slow variable. The bursting pattern evolves due to oscillations around stable and/or unstable foci of the fast subsystem. The Point-Point bursting oscillations can be further specified with regard to the two main bifurcations, causing the transition from silent to active phase and vice versa. For Point-Point bursting $\mathrm{Ca}^{2+}$ oscillations, we found Fold-Fold and SubHopf-SubHopf type of bursting.

3.1.1.1. Point-Point bursting of Fold-Fold type. In Fig. 1a and b two examples of the Fold-Fold type of Point-Point bursting are presented, which we found in models proposed by Shen and Larter [28] and Marhl et al. [30,46] (see Model 1 and the simplified Model 3 in Appendix A, respectively). By comparing Fig. 1a and b we see that in both cases the active phase of bursting starts and ends with a fold bifurcation (denoted by an up-triangle). We gain better understanding of the bursting pattern if we follow the trajectories depicted in Fig. 1a and b in the anti-clockwise direction starting at the lower stable steady state branch (marked by an arrow). As time progresses, the lower stable steady state branch loses stability via fold bifurcation, which sends the trajectory to the upper stable steady state branch, resulting in the first spike of $C a_{\mathrm{cyt}}$. The same scenario repeats for the upper stable steady state branch, with the difference that before the trajectory reaches the lower steady state branch it makes an additional return to the upper steady state. This results in a second spike of $C a_{\mathrm{cyt}}$. Finally, the attraction of the lower stable steady state closes the trajectory and the silent phase of bursting starts again.

3.1.1.2. Point-Point bursting of SubHopf-SubHopf type. The second example of Point-Point bursting is SubHopfSubHopf bursting [17], which can be found in the model proposed by Borghans et al. [29] (see Model 2 in Appendix A). The main characteristic of this type of bursting is that the active phase of bursting starts and ends with a subcritical Hopf bifurcation (denoted by a circle in Fig. 2). To explain the pattern of SubHopf-SubHopf bursting, we start to follow the trajectory in the anti-clockwise direction at the upper unstable steady state branch (marked by an arrow in Fig. 2). As time progresses the trajectory slowly diverges from the upper unstable branch and starts to unfold to the lower stable branch, thereby starting the bursting pattern. The trajectory passes the lower stable branch very quickly (lower arc of the trajectory) and starts to wrap towards the upper stable branch. The large amplitude of $C a_{\mathrm{cyt}}$ is damped due to high attractive stable foci on the upper stable branch and the trajectory settles near to the branch rather quickly. 

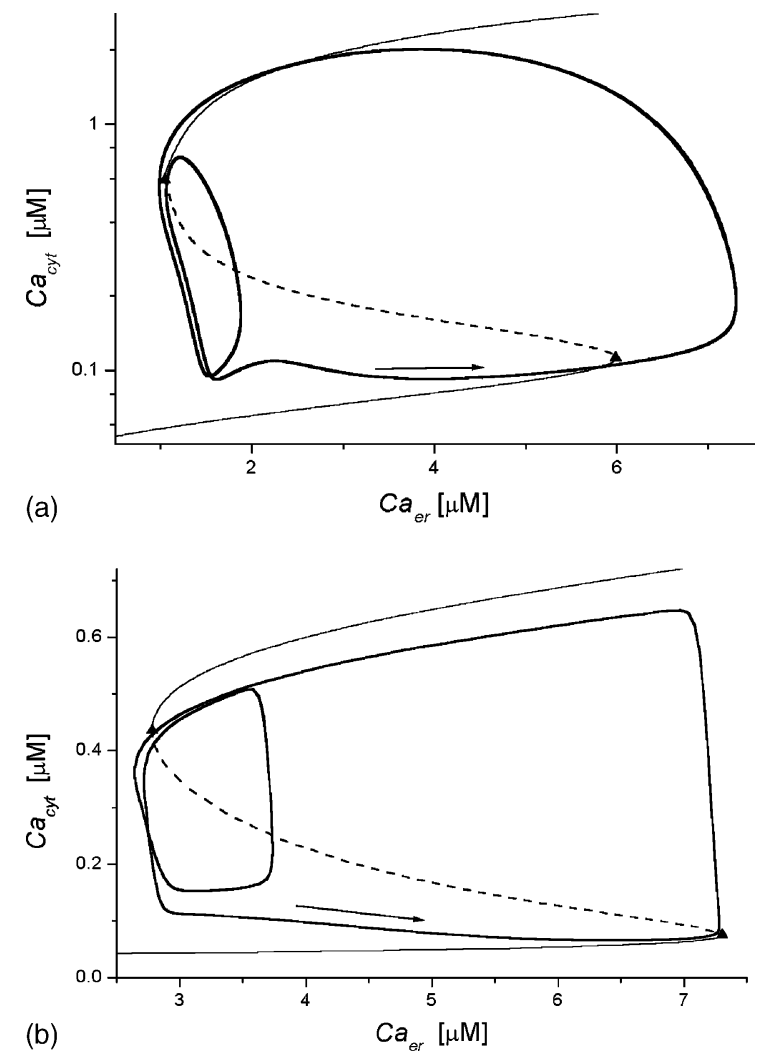

Fig. 1. Bifurcation diagram of the fast subsystem (only $C a_{\text {cyt }}$ is depicted), whereas the slow variable $\left(C a_{\text {er }}\right)$ is used as the bifurcation parameter. Solid (dashed) lines represent stable (unstable) steady states. Up-triangles denote fold bifurcations. The thick solid line represents the $2 \mathrm{D}$ projection of the trajectory in the whole phase space. (a) Results for the mathematical model proposed by Shen and Larter [28] (see Model 1 in Appendix A, $r=0.60$ ). (b) Results for the mathematical model proposed by Marhl et al. [30] (see the simplified Model 3 in Appendix A, $k_{\mathrm{ch}}=600 \mathrm{~s}^{-1}$ ).

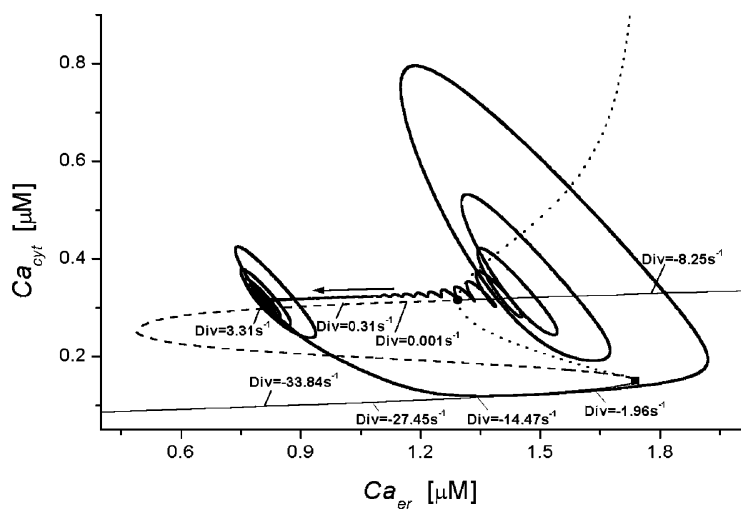

Fig. 2. Bifurcation diagram of the fast subsystem (only $C a_{\text {cyt }}$ is depicted), whereas the slow variable $\left(C a_{\text {er }}\right)$ is used as the bifurcation parameter. Solid (dashed) lines represent stable (unstable) steady states. Doted lines represent unstable periodic solutions. Circle (square) represents the subcritical Hopf (saddle homoclinic orbit) bifurcation. The thick solid line represents the 2D projection of the trajectory in the whole phase space. Numbers added to the bifurcation diagram represent the divergence of the fast subsystem at different points. Results are calculated for the mathematical model proposed by Borghans et al. [29] (see Model 2 in Appendix A, $r=1.13$ ). 
By passing the subcritical Hopf bifurcation the loop completes and the silent phase of bursting starts again as shown in Fig. 2. The square denotes the saddle homoclinic orbit bifurcation, which arises when the fold bifurcation of the lower stable steady state branch coincidences with the lower unstable periodic branch originating from the subcritical Hopf bifurcation. The saddle homoclinic orbit bifurcation does not influence the shape of the bursting pattern, but is nevertheless important, since it prevents the unstable periodic branch to turn stable. Therefore, this bursting is of the Point-Point and not of the Point-Cycle type.

In Fig. 2 the important phenomenon of the so-called slow passage effect $[12,13,17,47,48]$ can be well observed. This phenomenon enables the SubHopf-SubHopf bursting and can be explained as follows: Just before passing the subcritical Hopf bifurcation (see Fig. 2) in the anti-clockwise direction, the system is close to the rest state (silent phase of bursting). When the trajectory slowly passes through the bifurcation point, it remains close to the rest state despite the fact that the steady state had turned unstable (dashed line in Fig. 2). After a while, the trajectory diverges from the unstable foci and starts to unfold towards the lower stable steady state. The trajectory persists in close proximity of the unstable branch due to the slow passage effect, which can be well explained by the divergence of the lower stable and upper unstable steady state branch. The values of divergence express the attractive/repelling properties of the steady states. Some characteristic values of the divergence, important for the behaviour of the trajectory, are added to the bifurcation diagram in Fig. 2. The divergence of the upper unstable steady state is just slightly above zero for an extensive amount of time after the trajectory passes the subcritical Hopf bifurcation, where the divergence in 2D fast subsystem is exactly zero. Hence, the unstable branch is weakly repellent in a large parameter range of the bifurcation diagram. Therefore, the trajectory stays close to the unstable branch despite the fact that it is unstable. When the divergence reaches a large enough positive value, the trajectory starts to unfold from the unstable branch considerably. Moreover, the divergence of the lower steady state is much more negative at this stage as it is directly below the subcritical Hopf bifurcation, which additionally contributes to the ending of the silent phase.

\subsubsection{Point-Cycle bursting}

The Point-Cycle bursting can also be well studied by the fast-slow burster analysis (see Section 2). The characteristic of the Point-Cycle bursting is that active and silent phases of bursting depend on a stable steady state (Point) as well as on a stable limit cycle (Cycle) in phase space of the fast subsystem. We give three examples of planar (the fast subsystem is two-dimensional) Point-Cycle bursters, i.e.: Fold-Hopf bursting via Fold-Fold hysteresis loop, Hopf-Hopf bursting, and SubHopf-SubHopf bursting, which can be found in models proposed by Marhl et al. [30], and Kummer et al. [31].

3.1.2.1. Point-Cycle bursting of Fold-Hopf type via Fold-Fold hysteresis loop. The first example of Point-Cycle bursting is the Fold-Hopf bursting via Fold-Fold hysteresis loop, which appears in the mathematical model for $\mathrm{Ca}^{2+}$ oscillations proposed by Kummer et al. [31] (see Model 4 in Appendix A). This type of bursting has already been analysed previously by Brusch et al. [41]. The behaviour of the Fold-Hopf bursting via Fold-Fold hysteresis loop, shown in Fig. 3 , can be explained by use of the fast-slow burster analysis. We start to follow the trajectory in the anti-clockwise direction at the silent phase of bursting (marked by an arrow in Fig. 3). The silent phase of bursting ends with a fold bifurcation (denoted by an up-triangle). The active phase of fast oscillations with decreasing amplitude begins when the

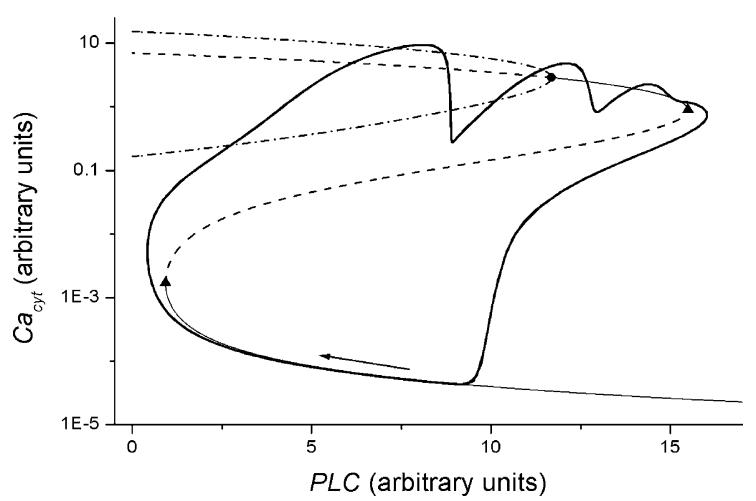

Fig. 3. Bifurcation diagram of the fast subsystem (only $C a_{\text {cyt }}$ is depicted), whereas the slow variable $(P L C)$ is used as the bifurcation parameter. Solid (dashed) lines represent stable (unstable) steady states. Dash-doted lines represent stable periodic solutions. Uptriangles denote the two fold bifurcations. Circle represents the supercritical Hopf bifurcation. The thick solid line represents the 2D projection of the trajectory in the whole phase space. Results are calculated for the mathematical model proposed by Kummer et al. [31] (see Model 4 in Appendix A, $k_{2}=2.8$ ). 
trajectory moves upwards to the branch of unstable foci with corresponding stable limit cycles. The amplitudes of bursting oscillations become even smaller by approaching to the supercritical Hopf bifurcation (denoted by a circle in Fig. 3). The transition to the silent phase of bursting starts again, when the trajectory passes the supercritical Hopf bifurcation. However, this transition does not happen abruptly since the eigen values of the stable foci in addition to their negative real parts also have imaginary parts. Consequently, the trajectory settles down to the stable foci branch in form of damped oscillations, which slightly prolongs the active phase of bursting. Finally, after passing the second fold bifurcation (denoted by an up-triangle) the trajectory moves downward to the stable branch again and herewith completes one period of bursting.

3.1.2.2. Point-Cycle bursting of Hopf-Hopf type. Another example of Point-Cycle bursting is the Hopf-Hopf bursting, which can be found in the model proposed by Marhl et al. [30] (see the simplified Model 3 in Appendix A). The HopfHopf bursting is studied by the fast-slow burster analysis (Fig. 4). Like in the previous cases, we start to explain the system behaviour at the silent phase of bursting (marked by an arrow in the anti-clockwise direction). As time progresses the trajectory passes the supercritical Hopf bifurcation (denoted by a circle in Fig. 4). Thereby the stable steady state branch turns unstable and after a while, the trajectory starts to unfold towards the stable periodic branches originating from the supercritical Hopf bifurcation. This starts the active phase of bursting. Note that like in the previously discussed SubHopf-SubHopf type of Point-Point bursting here the slow passage effect is present as well. Due to the slow passage effect the trajectory remains close to the rest state even after passing the bifurcation point. This behaviour can be well explained by calculating the attracting/repelling properties of steady states and limit cycles in the fast $2 \mathrm{D}$ subsystem. To this purpose, the values of divergence are added to the bifurcation diagram (see Fig. 4). The divergence of the unstable foci is just slightly above zero for an extensive amount of time after the trajectory passes the supercritical Hopf bifurcation. Hence, the unstable branch is weakly repellent and the trajectory stays near it. In addition, close to the supercritical Hopf bifurcation the trajectory is only weekly attracted by the stable periodic states since their divergence is just slightly negative. When the trajectory moves further along the unstable branch, it becomes increasingly repellent (divergence increases) and the stable periodic branches become more and more attractive (divergence decreases). Both effects result in forcing the trajectory to unfold to the stable limit cycle. However, the trajectory does not stay in the stable periodic regime for a longer time but makes a rather rapid turn to the stable steady state on the other side of the Hopf bifurcation. Oscillations around the stable branch are damped due to attractive stable foci (the divergence is negative). Herewith, the silent phase of bursting starts again as shown in Fig. 4.

3.1.2.3. Point-Cycle bursting of SubHopf-SubHopf type. The last example of Point-Cycle bursting is the SubHopfSubHopf bursting, which can be found in the model proposed by Marhl et al. [30] (see Model 3 in Appendix A). The name SubHopf-SubHopf bursting has already been used for a type of a Point-Point burster in Section 3.1.1. However, in case of the Point-Cycle bursting type the fast subsystem has a limit cycle instead of the second point attractor. The bifurcation diagram for the SubHopf-SubHopf type of Point-Cycle bursting (Fig. 5) differs considerably from the previously analysed SubHopf-SubHopf type of Point-Point bursting (Fig. 2, Section 3.1.1). The most important dif-

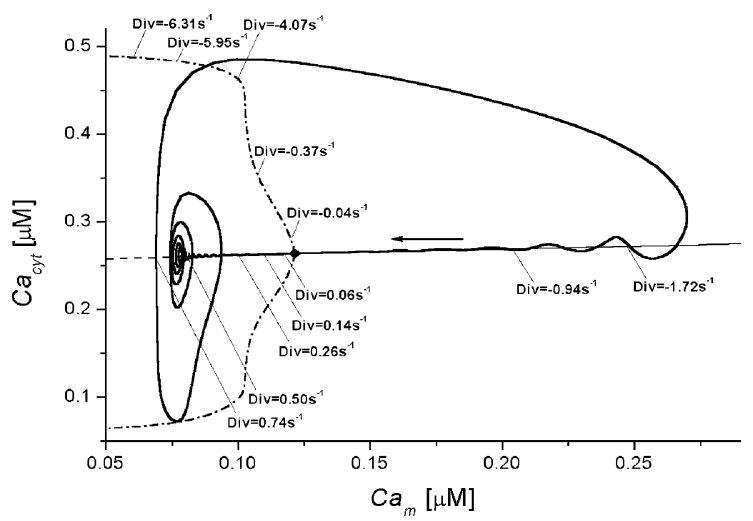

Fig. 4. Bifurcation diagram of the fast subsystem (only $C a_{\text {cyt }}$ is depicted), whereas the slow variable $\left(C a_{m}\right)$ is used as the bifurcation parameter. Solid (dashed) lines represent stable (unstable) steady states. Dash-doted lines represent stable periodic solutions. Circle represents the supercritical Hopf bifurcation. The thick solid line represents the $2 \mathrm{D}$ projection of the trajectory in the whole phase space. Numbers added to the bifurcation diagram represent the divergence of the fast subsystem at different points. Results are calculated for the mathematical model proposed by Marhl et al. [30] (see the simplified Model 3 in Appendix A, $k_{\mathrm{ch}}=4332 \mathrm{~s}^{-1}$ ). 


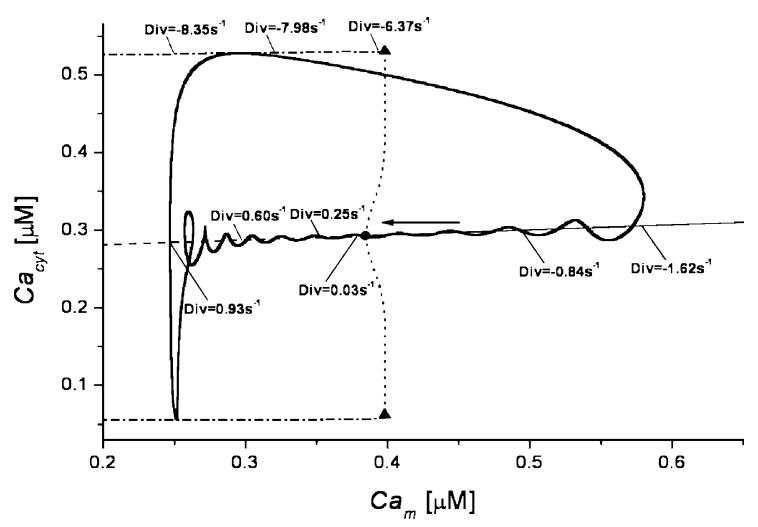

Fig. 5. Bifurcation diagram of the fast subsystem (only $C a_{\text {cyt }}$ is depicted), whereas the slow variable $\left(C a_{m}\right)$ is used as the bifurcation parameter. Solid (dashed) lines represent stable (unstable) steady states. Dash-doted (doted) lines represent stable (unstable) periodic solutions. Circle (up-triangle) represents the subcritical Hopf (fold limit cycle) bifurcation. The thick solid line represents the 2D projection of the trajectory in the whole phase space. Numbers added to the bifurcation diagram represent the divergence of the fast subsystem at different points. Results are calculated for the mathematical model proposed by Marhl et al. [30] (see Model 3 in Appendix $\mathrm{A}, k_{\mathrm{ch}}=3500 \mathrm{~s}^{-1}$ ).

ference is that here in case of the Point-Cycle bursting we do not have the saddle homoclinic orbit bifurcation anymore. Therefore, the unstable periodic branches originating form the subcritical Hopf bifurcation change their stability via a fold limit cycle bifurcation (denoted by an up-triangle in Fig. 5). The arising stable periodic branches are of key importance for the Point-Cycle bursting, whereas the fold limit cycle bifurcation itself does not influence the bursting pattern directly.

The SubHopf-SubHopf type of Point-Cycle bursting can be explained by the fast-slow burster analysis (Fig. 5). We start at the silent phase of bursting (marked by an arrow in the anti-clockwise direction). When the trajectory passes the subcritical Hopf bifurcation, (denoted by a circle in Fig. 5) the stable steady state branch loses its stability and turns unstable. Therefore, the trajectory slowly starts to unfold from the unstable steady states to stable periodic attractors. However, it takes a considerable time before the trajectory reaches the stable limit cycle. This indicates that the slow passage effect is present. The trajectory stays close to the rest state even after passing the subcritical Hopf bifurcation. The divergence of the fast $2 \mathrm{D}$ subsystem gives a deeper insight into this phenomenon. Calculated values of the divergence are added to the bifurcation diagram (see Fig. 5). We see that after the trajectory passes the subcritical Hopf bifurcation the unstable steady state branch is weakly repellent (the positive value of divergence is close to zero). Therefore, the trajectory stays near the unstable periodic branch for a considerable amount of time and consequently the transition to the stable limit cycle is delayed. As time progresses the unstable periodic branch gets increasingly repellent (divergence increases), which speeds up the transition to the stable limit cycle. This is accompanied by the increasing attractive properties of limit cycles (divergence decreases), which additionally contributes to the ending of the silent phase. The stable periodic regime is abandoned by the rapid turn to the stable steady state on the other side of the subcritical Hopf bifurcation. Oscillations around the stable branch are damped due to attractive stable foci (the divergence is negative). The silent phase of bursting starts again.

\subsection{Quasi-periodic bursting}

Quasi-periodicity is a rather rare phenomenon in the mathematical models for calcium oscillations. Only in the model proposed by Borghans et al. [29], a relatively narrow window of quasi-periodic behaviour can be found. We prove the quasi-periodic behaviour by calculating the first and the second Lyapunov exponent [45], which are both zero in this case.

Quasi-periodic $\mathrm{Ca}^{2+}$ oscillations in the model [29] (see Model 2 in Appendix A) are analysed by the fast-slow burster analysis (see Fig. 6). The bifurcation diagram in Fig. 6 is very similar to that obtained in Fig. 2, which was carried out for the same mathematical model [29]. The main difference between the two diagrams is the existence of stable periodic branches in Fig. 6, which evolve by changing the stability of the unstable periodic branches that originate from the subcritical Hopf bifurcation. The two stable periodic branches lose their stability via the second fold limit cycle bifurcation, resulting in a new pair of unstable periodic branches, from which the lower one collides with the fold 


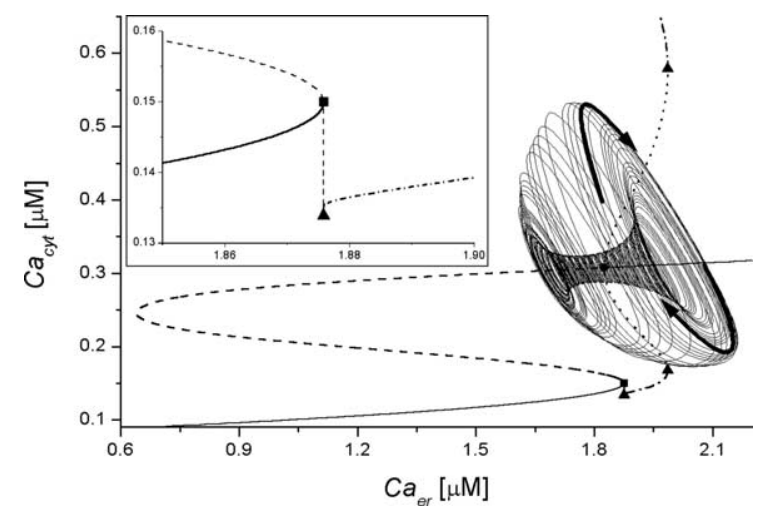

Fig. 6. Bifurcation diagram of the fast subsystem (only $C a_{\text {cyt }}$ is depicted), whereas the slow variable $\left(C a_{\text {er }}\right)$ is used as the bifurcation parameter. Solid (dashed) lines represent stable (unstable) steady states. Dash-doted (doted) lines represent stable (unstable) periodic solutions. Circle (up-triangle) represents the subcritical Hopf (fold limit cycle) bifurcation. Square denotes the homoclinic bifurcation with a homoclinic torus structure. The thick solid line represents the 2D projection of the trajectory in the whole phase space. Results are calculated for the mathematical model proposed by Borghans et al. [29] (see Model 2 in Appendix A, $r=0.99$ ).

bifurcation of the lower stable steady state branch (see insert in Fig. 6). Herewith, a homoclinic bifurcation (denoted by a square) with a homoclinic torus structure comes into existence. From this ascertainment, we may conclude that the torus attractor appears via fold limit cycle bifurcation with a homoclinic torus structure [17].

For better understanding of the complex bursting behaviour in Fig. 6 we start to follow the trajectory in the clockwise direction starting at high amplitude oscillations on the right side of the torus (marked by a pair of arrows). As time progresses the trajectory is drawn towards the stable steady state, which results in oscillations of even smaller amplitudes. When the subcritical Hopf bifurcation is exceeded, the former stable steady state becomes unstable and the trajectory starts to unfold to the stable periodic branch. However, the trajectory never reaches the stable periodic branch since it always passes on the other side of the fold limit cycle bifurcation (denoted with an up-triangle), where it is again caught by the attraction of the upper steady states. They are the only stable attractors in that region of the phase space. This procedure repeats again and again, nevertheless with the small but crucial difference, that the trajectory diverts away from the stable periodic branches upwards at always-different moments (see Fig. 6). Consequently, the trajectory never closes in on itself and the torus attractor evolves.

\subsection{Chaotic bursting}

All four examined mathematical models exhibit chaotic behaviour in a more or less wide range of the oscillatory regime. We proved the chaotic behaviour by calculating the largest Lyapunov exponent (see Section 2). For a more detailed analysis of parameter values and regions in which chaos occurs in a particular model, we refer the reader to articles in which the mathematical models were originally published [28-31].

Chaotic bursting oscillations can be classified in the same way as regular bursting oscillations e.g.: Point-Point or Point-Cycle bursting. That means, that we can obtain the same bifurcation diagrams with the fast-slow burster analysis as we did for the regular periodic oscillations. Nevertheless, we cannot use this analysis to explain the complex irregular bursting patterns because the fast subsystem fails to reflect the chaotic behaviour, since it is only two-dimensional. Therefore, we classify different chaotic bursting patterns accordingly to the transition type from regular to chaotic behaviour. We distinguish two different roots to chaos i.e. period doubling and intermittency, which reflect two different bursting behaviours.

\subsubsection{Period doubling}

The period doubling root is a common transition to chaos and is present in all four examined models [28-31]. As an example, we show the bifurcation diagram of the mathematical model proposed by Shen and Larter [28] in Fig. 7a. The period doubling root to chaos can be explained as follows: A stable periodic branch turns unstable and additionally splits into two new stable periodic branches which again split into two new stable periodic branches and so on (see Fig. 7a), thereby creating the vast multitude of frequency as well as amplitude windows which characterize chaotic behaviour. Fig. $7 \mathrm{~b}$ shows a typical time course of chaotic bursting, which appears through a sequence of period doubling 

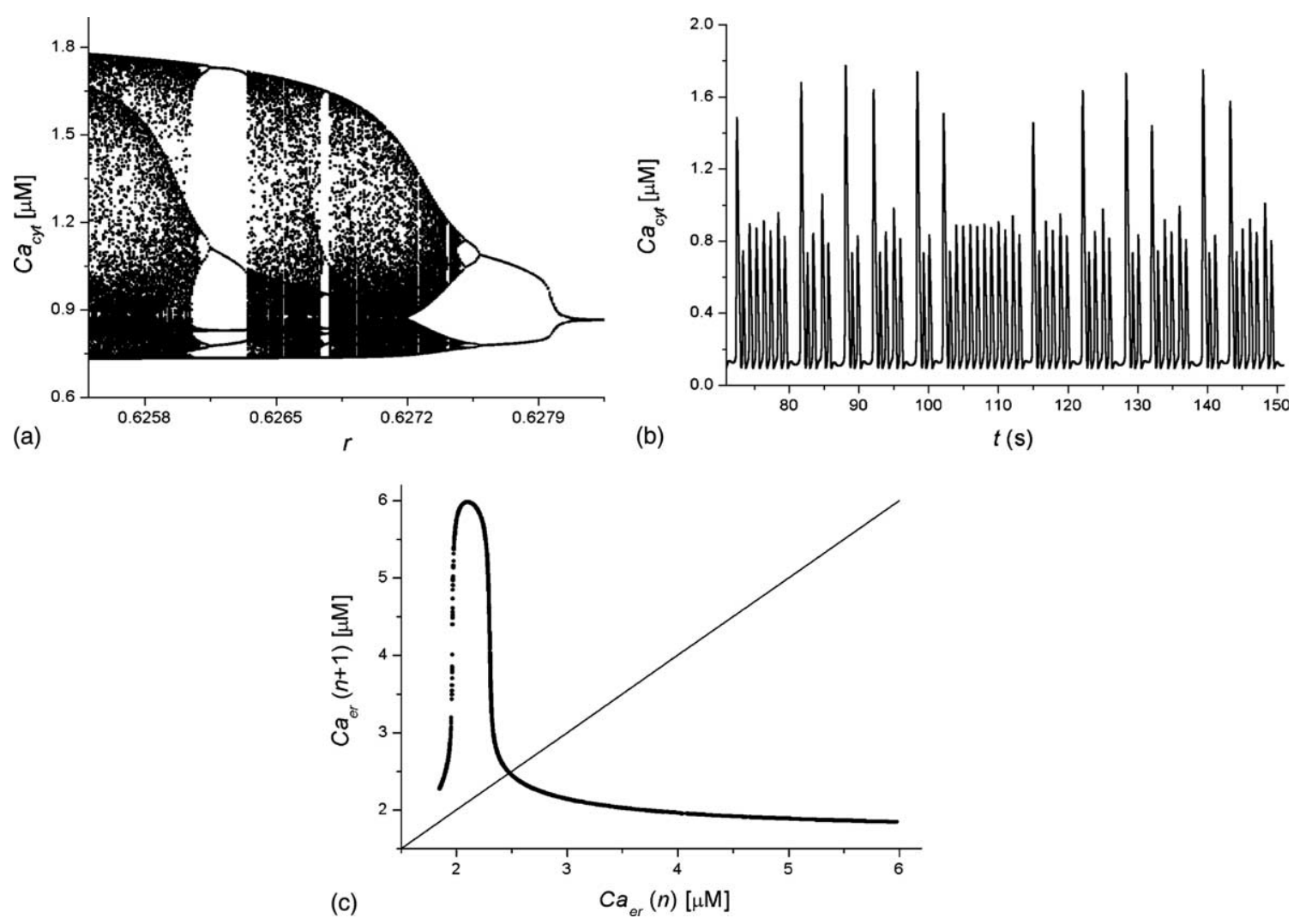

Fig. 7. Analysis of the chaotic $\mathrm{Ca}^{2+}$ bursting for the mathematical model proposed by Shen and Larter [28] (see Model 1 in Appendix A). (a) Bifurcation diagram $C a_{\text {cyt }}$ versus $r$. (b) Time course of $C a_{\text {cyt }}$ for $r=0.6258$. (c) Return map of $C a_{\mathrm{er}}$.

bifurcations. The time course of $C a_{\text {cyt }}$ in Fig. $7 \mathrm{~b}$ is calculated for $r=0.6258$ in the model proposed by Shen and Larter [28]. It clearly reflects the high diversity of frequencies as well as amplitudes, which is a consequence of the period doubling root to chaos. This can also be seen in the return map, which is presented in Fig. 7c. In this figure, the successive maxima of $C a_{\mathrm{er}}$ are plotted against their predecessors. If these maxima are plotted for a very long time series, a continuous curve is obtained, which is a strong evidence for deterministic chaos [49].

\subsubsection{Intermittency}

The intermittent root to chaos is not very common and slightly more entangled than the period doubling root. We found the intermittent root to chaos in the models proposed by Shen and Larter [28] and Marhl et al. [30]. The bifurcation diagram presented in Fig. 8a was calculated for the mathematical model proposed by Marhl et al. [30]. It shows the characteristic intermittent transition from simple regular oscillations to chaotic behaviour. For $k_{\mathrm{ch}}=2850 \mathrm{~s}^{-1}$ the time course of intermittent chaotic bursting is presented in Fig. 8b. Note that the amplitudes of the main $C a_{\text {cyt }}$ spikes remain nearly constant all the time. The corresponding return map to the time course of Fig. 8b is shown in Fig. 8c. In the return map, the successive maxima of $C a_{m}$ are plotted against their predecessors. Like in the Fig. 7c an almost continuous curve is obtained, which represents strong evidence of deterministic chaos. Nevertheless, there is an important difference between Figs. 7c and 8c, which contributes to distinct corresponding bursting patterns shown in Figs. $7 \mathrm{~b}$ and $8 \mathrm{~b}$, respectively. The insert in Fig. 8c shows that points of the return map in Fig. $8 \mathrm{c}$ come very close to the bisector line. Points that form the bisector line represent cycles of constant amplitudes, since there each maximum has the same amplitude as its predecessor. Consequently, the cycles of the trajectory, which are represented by points near the bisector line, maintain a very similar form (see Fig. 8b). Complex behaviour evolves because the trajectory leaves the points near the bisector line intermittently, that is at always-different moments, which makes the long time behaviour chaotic [40]. 

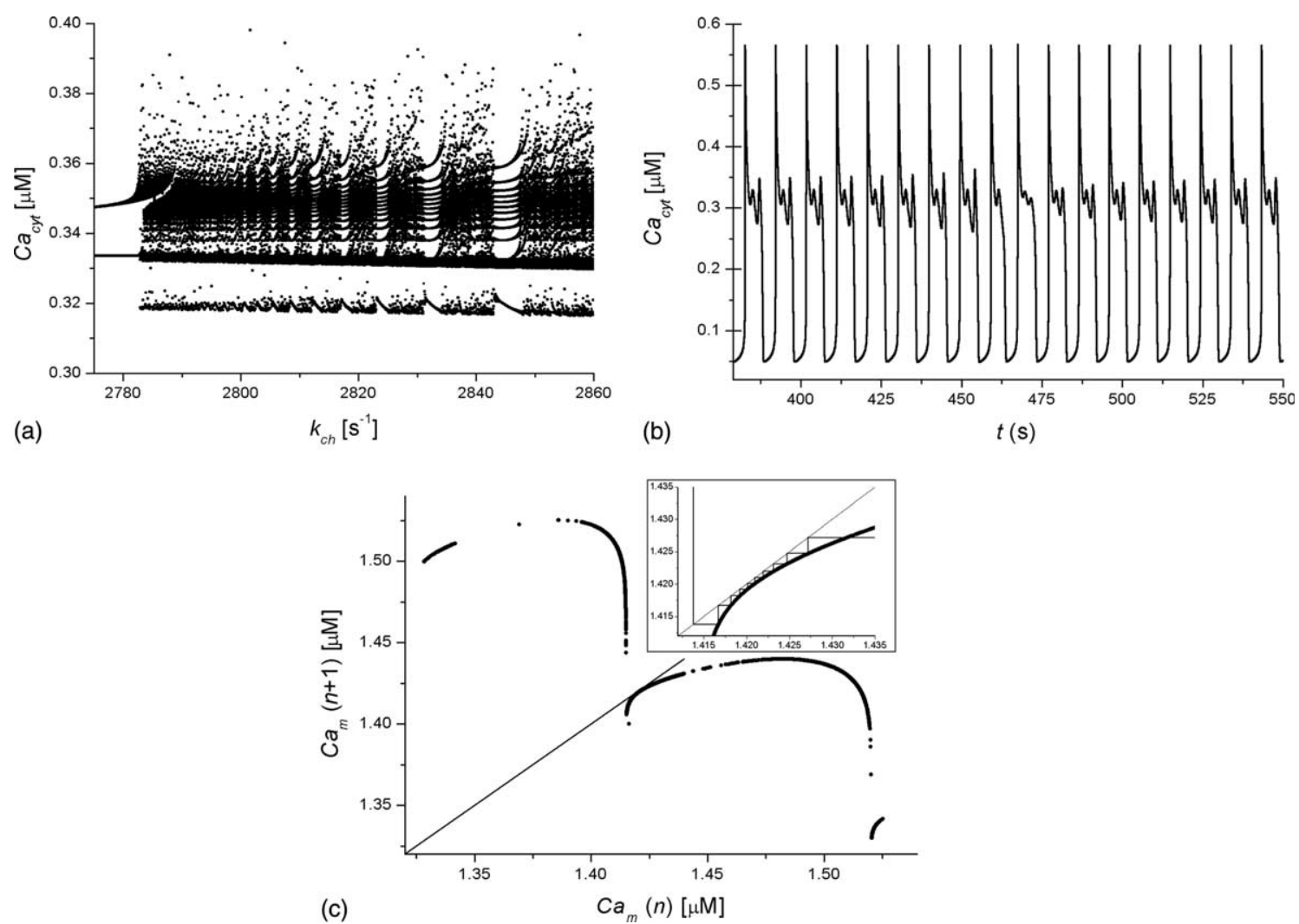

Fig. 8. Analysis of the chaotic regime for the mathematical model proposed by Marhl et al. [30] (see Model 3 in Appendix A). (a) Bifurcation diagram of $C a_{\mathrm{cyt}}$ versus $k_{\mathrm{ch}}$. (b) Time course of $C a_{\mathrm{cyt}}$ for $k_{\mathrm{ch}}=2850 \mathrm{~s}^{-1}$. (c) Return map of $C a_{m}$.

\section{Discussion}

In the paper, bursting $\mathrm{Ca}^{2+}$ oscillations in non-excitable cells are studied for four recently proposed mathematical models [28-31]. Separately, we analyse regular, quasi-periodic and chaotic $\mathrm{Ca}^{2+}$ bursting oscillations. The main bursting types are further classified into several subtypes. Results indicate that regular Point-Point bursting is very common in mathematical models for intracellular $\mathrm{Ca}^{2+}$ oscillations. Fold-Fold subtype of Point-Point bursting was found in the models proposed by Shen and Larter [28] and Marhl et al. [30], whereas SubHopf-SubHopf subtype of Point-Point bursting was found in the model proposed by Borghans et al. [29]. Furthermore, we also found three different types of regular Point-Cycle bursting. Fold-Hopf via Fold-Fold hysteresis loop subtype of Point-Cycle bursting was found in the model proposed by Kummer et al. [31]. Additionally, we identified Hopf-Hopf and SubHopf-SubHopf subtype of Point-Cycle bursting in the model proposed by Marhl et al. [30]. However, the Cycle-Cycle type of bursting cannot be found in the models describing intracellular $\mathrm{Ca}^{2+}$ oscillations in non-excitable cells. This type of bursting is well known for the Hodgin-Huxley-like models, which describe electrical excitations in nerve cells [50-52]. The main characteristic of the Cycle-Cycle bursting, expressed in terms of fast-slow burster analysis, is that the active bursting phase corresponds to a limit cycle in the fast subsystem and the silent phase is linked to another limit cycle with a small amplitude (subthreshold) oscillation.

Furthermore, besides the regular bursting patterns we also found more entangled bursting behaviour of quasi-periodic and chaotic type. Quasi-periodicity was found in the mathematical model proposed by Borghans et al. [29]. Our results show that the torus attractor appears via fold limit cycle bifurcation with a homoclinic torus structure. Chaotic bursting was identified in all four examined mathematical models [28-31]. We distinguish two types of chaotic bursting according to the transition root from regular to complex chaotic behaviour. The period doubling root to chaos was found in all examined models, whereas intermittency was found in the models proposed by Shen and Larter [28] and Marhl et al. [30]. In the latter model the intermittent root to chaos was already described earlier by Haberichter et al. [40]. 
The biological importance of bursting oscillations is well established. Bursting behaviour was found to be the primary mode of information processing and signal transduction in many biological systems, like for example in different neuron types, pancreatic $\beta$-cells and hepatocytes [1-9,24-27]. It has been shown that biologically relevant information of $\mathrm{Ca}^{2+}$ oscillations is primarily frequency encoded [46,53-58]. However, some studies also emphasize the importance of amplitude encoding [59,60]. From this point of view, it is important to analyse different bursting types of $\mathrm{Ca}^{2+}$ oscillations, since different types of bursting behaviour could reflect different encoding of biologically relevant information.

Another important biological aspect of studying different bursting types is that this could further improve our understanding of intercellular $\mathrm{Ca}^{2+}$ signalling, in particular, cell coupling and the phenomenon of synchronisation. In some other systems, it has been shown that bursters incorporating the slow passage effect can synchronize almost instantaneously $[17,18,61]$. This is of important biological meaning, since fast and reliable cell synchronisation in signal transduction is crucial for flawless functioning of living organisms. From the mathematical point of view, the very fast synchronization of bursters expressing slow passage effect can be well explained. The slow passage phase represents the most flexible part of the trajectory and it can be therefore shortened significantly by weak input from other bursters. This property provides a powerful mechanism for instantaneous synchronization of bursters even when they have essentially different interburst frequencies. From Figs. 2, 4 and 5 it can be well observed that the trajectory spends approximately half of the quiescent time near the unstable foci branch. During this time, the system is ready to fire in response to the very first pulse coming from the neighbouring burster. As the result, instantaneous synchronization occurs in a very short time. Usually, the synchronization occurs already after the first spike [18,61]. Our preliminary results on this topic, concerning intracellular $\mathrm{Ca}^{2+}$ oscillations, confirm this completely. However, the influence of different types of bursting $\mathrm{Ca}^{2+}$ oscillations on the synchronisation properties of the system should be further studied and will be presented in more detail in a separate paper.

Complex bursting patterns also seem to be of special biological importance. Chaos is present in several biological systems like in cardiac rhythms, for example [62]. In particular, the intermittent chaotical behaviour seems to be of special advantage in biological systems. Intuitively, the high biological importance of intermittency can be well explained. Intermittency incorporates a quasi-order rhythm, which is predominantly present in a biological system [63], as well as irregularities, which could also play a role in real life. Some studies suggest that this irregular chaotic behaviour is important, because it assures high sensitivity and flexibility of biological systems [64,65]. However, this cannot be generalised. In our previous studies, for example, we showed that regular periodic oscillators can also be highly sensitive and flexible in response to external perturbations [66]. Therefore, further studies will be necessary to determine more precisely the biological role of different types of complex $\mathrm{Ca}^{2+}$ oscillations.

\section{Appendix A}

In Appendix A differential equations for all four examined mathematical models [28-31] are presented as well as the parameter values for which all calculations were made.

\section{A.1. Model 1}

The mathematical model proposed by Shen and Larter [28] is described by the following differential equations:

$$
\begin{aligned}
& \frac{\mathrm{d} C a_{\mathrm{cyt}}}{\mathrm{d} t}=J_{\mathrm{ch}}+J_{\text {leak }}-J_{\text {pump }}+J_{\text {in }}-J_{\text {out }}, \\
& \frac{\mathrm{d} C a_{\mathrm{er}}}{\mathrm{d} t}=J_{\text {pump }}-J_{\mathrm{ch}}-J_{\text {leak }}, \\
& \frac{\mathrm{d} I P_{3}}{\mathrm{~d} t}=J_{+}-J_{-},
\end{aligned}
$$

where

$$
\begin{aligned}
& J_{\mathrm{ch}}=k_{\mathrm{ch}}\left(\frac{I P_{3}^{4}}{I P_{3}^{4}+K_{1}^{4}}\right)\left(\frac{K_{4} C a_{\mathrm{cyt}}}{\left(C a_{\mathrm{cyt}}+K_{4}\right)\left(C a_{\mathrm{cyt}}+K_{5}\right)}\right)^{3} C a_{\mathrm{er}}, \\
& J_{\text {leak }}=k_{\text {leak }} C a_{\mathrm{er}},
\end{aligned}
$$




$$
\begin{aligned}
& J_{\text {pump }}=k_{\text {pump }} \frac{C a_{\mathrm{cyt}}^{2}}{C a_{\mathrm{cyt}}^{2}+K_{2}^{2}}, \\
& J_{\text {in }}=k_{\text {in } 1} \cdot r+k_{\mathrm{in} 2}, \\
& J_{\text {out }}=k_{\text {out }} C a_{\mathrm{cyt}}, \\
& J_{+}=k_{+} \cdot r \frac{C a_{\mathrm{cyt}}}{C a_{\mathrm{cyt}}+K_{3}}, \\
& J_{-}=k_{-} \cdot I P_{3} .
\end{aligned}
$$

Parameters for which all calculations are made if not otherwise stated: $k_{\mathrm{ch}}=3000.0 \mu \mathrm{M} \mathrm{s}^{-1}, k_{\text {leak }}=1.0 \mathrm{~s}^{-1}, k_{\mathrm{pump}}=50.0$ $\mu \mathrm{M} \mathrm{s}^{-1}, k_{\text {in } 1}=4.0 \mu \mathrm{M} \mathrm{s}^{-1}, k_{\text {in } 2}=1.0 \mu \mathrm{M} \mathrm{s}^{-1}, k_{\text {out }}=10.0 \mathrm{~s}^{-1}, k_{+}=4.0 \mu \mathrm{M} \mathrm{s}^{-1}, k_{-}=2.0 \mu \mathrm{M} \mathrm{s}^{-1}, K_{1}=K_{2}=0.2 \mu \mathrm{M}$, $K_{3}=1.0 \mu \mathrm{M}, K_{4}=K_{5}=0.69 \mu \mathrm{M}, r=0.2345-0.6859$.

\section{A.2. Model 2}

The mathematical model proposed by Borghans et al. [29] is described by the following differential equations:

$$
\begin{aligned}
& \frac{\mathrm{d} C a_{\mathrm{cyt}}}{\mathrm{d} t}=J_{\text {in }}+J_{\text {leak }}-J_{\text {pump }}+J_{\text {er }}-J_{\text {out }}, \\
& \frac{\mathrm{d} C a_{\mathrm{er}}}{\mathrm{d} t}=J_{\text {pump }}-J_{\text {er }}-J_{\text {leak }}, \\
& \frac{\mathrm{d} I P_{3}}{\mathrm{~d} t}=J_{A}-J_{D}-J_{C}
\end{aligned}
$$

where

$$
\begin{aligned}
& J_{\text {in }}=k_{\text {in } 1} \cdot r+k_{\text {in } 2} \\
& J_{\text {leak }}=r \cdot k_{\text {leak }} \frac{I P_{3}^{4}}{I P_{3}^{4}+K_{a}^{4}} \cdot \frac{C a_{\mathrm{er}}^{2}}{C a_{\mathrm{er}}^{2}+K_{y}^{2}} \cdot \frac{C a_{\mathrm{cyt}}^{4}}{C a_{\mathrm{cyt}}^{4}+K_{z}^{4}}, \\
& J_{\text {pump }}=k_{\mathrm{pump}} \frac{C a_{\mathrm{cyt}}^{2}}{C a_{\mathrm{cyt}}^{2}+K_{2}^{2}}, \\
& J_{\text {out }}=k_{\mathrm{out}} C a_{\mathrm{cyt}}, \\
& J_{\mathrm{er}}=k_{f} C a_{\mathrm{er}}, \\
& J_{A}=r \cdot k_{p}, \\
& J_{D}=k_{d} \frac{I P_{3}^{2}}{I P_{3}^{2}+K_{p}^{2}} \cdot \frac{C a_{\mathrm{cyt}}^{4}}{C a_{\mathrm{cyt}}^{4}+K_{d}^{4}}, \\
& J_{C}=\varepsilon \cdot I P_{3} .
\end{aligned}
$$

Parameters for which all calculations are made if not otherwise stated: $k_{\text {leak }}=19.5 \mu \mathrm{M} \mathrm{min}^{-1}, k_{\text {pump }}=6.5 \mu \mathrm{M} \mathrm{min}{ }^{-1}$, $k_{\text {in } 1}=1.0 \mu \mathrm{M} \mathrm{min}^{-1}, k_{\text {in } 2}=2.0 \mu \mathrm{M} \mathrm{min}^{-1}, k_{p}=2.5 \mu \mathrm{M} \mathrm{min}^{-1}, k_{d}=80 \mu \mathrm{M} \mathrm{min}-1, k_{f}=1.0 \min ^{-1}, k_{\text {out }}=10 \mathrm{~min}^{-1}$, $K_{2}=0.1 \mu \mathrm{M}, K_{a}=0.4 \mu \mathrm{M}, K_{y}=0.2 \mu \mathrm{M}, K_{z}=0.3 \mu \mathrm{M}, K_{p}=1.0 \mu \mathrm{M}, K_{d}=0.4 \mu \mathrm{M}, \varepsilon=0.1, r=0.7981-1.196$.

\section{A.3. Model 3}

The mathematical model proposed by Marhl et al. [30] is described by the following differential equations:

$$
\begin{aligned}
& \frac{\mathrm{d} C a_{\mathrm{cyt}}}{\mathrm{d} t}=J_{\mathrm{ch}}-J_{\text {pump }}+J_{\text {leak }}+J_{\text {out }}-J_{\text {in }}+J_{C a P r}-J_{P r}, \\
& \frac{\mathrm{d} C a_{\mathrm{er}}}{\mathrm{d} t}=\frac{\beta_{\mathrm{er}}}{\rho_{\mathrm{er}}}\left(J_{\text {pump }}-J_{\mathrm{ch}}-J_{\text {leak }}\right),
\end{aligned}
$$




$$
\frac{\mathrm{d} C a_{m}}{\mathrm{~d} t}=\frac{\beta_{m}}{\rho_{m}}\left(J_{\text {in }}-J_{\text {out }}\right),
$$

where

$$
\begin{aligned}
& J_{\mathrm{ch}}=k_{\mathrm{ch}} \frac{C a_{\mathrm{cyt}}^{2}}{C a_{\mathrm{cyt}}^{2}+K_{1}^{2}}\left(C a_{\mathrm{er}}-C a_{\mathrm{cyt}}\right), \\
& J_{\mathrm{pump}}=k_{\mathrm{pump}} C a_{\mathrm{cyt}}, \\
& J_{\text {leak }}=k_{\text {leak }}\left(C a_{\mathrm{er}}-C a_{\mathrm{cyt}}\right), \\
& J_{P r}=k_{+} C a_{\mathrm{cyt}} P r \\
& J_{C a P r}=k_{-} C a P r \\
& J_{\text {in }}=k_{\mathrm{in}} \frac{C a_{\mathrm{cyt}}^{8}}{C a_{\mathrm{cyt}}^{8}+K_{2}^{8}}, \\
& J_{\mathrm{out}}=\left(k_{m} \frac{C a_{\mathrm{cyt}}^{2}}{C a_{\mathrm{cyt}}^{2}+K_{1}^{2}}+k_{\mathrm{mit}}\right) C a_{m}, \\
& P r_{\mathrm{tot}}=P r+C a P r, \\
& C a_{\mathrm{tot}}=C a_{\mathrm{cyt}}+\frac{\rho_{\mathrm{er}}}{\beta_{\mathrm{er}}} C a_{\mathrm{er}}+\frac{\rho_{m}}{\beta_{m}} C a_{m}+C a P r
\end{aligned}
$$

Parameters for which all calculations are made if not otherwise stated: $k_{\text {leak }}=0.05 \mathrm{~s}^{-1}, k_{\text {pump }}=20.0 \mathrm{~s}^{-1}, k_{\text {in }}=300$ $\mu \mathrm{M} \mathrm{s}^{-1}, k_{m}=125 \mathrm{~s}^{-1}, k_{\text {mit }}=0.00625 \mathrm{~s}^{-1}, k_{+}=0.1 \mu \mathrm{M} \mathrm{s}^{-1}\left(\right.$ Fig. $5 k_{+}=0.09 \mu \mathrm{M} \mathrm{s}^{-1}$ ), $k_{-}=0.01 \mathrm{~s}^{-1}, K_{1}=5.0 \mu \mathrm{M}$, $K_{2}=0.8 \mu \mathrm{M}, C a_{\mathrm{tot}}=90 \mu \mathrm{M}, P r_{\mathrm{tot}}=120 \mu \mathrm{M}, \rho_{\mathrm{er}}=0.01, \beta_{\mathrm{er}}=0.0025, \rho_{m}=0.01, \beta_{m}=0.0025, k_{\mathrm{ch}}=473.8-4473.4 \mathrm{~s}^{-1}$.

\section{A.3.1. Simplified Model 3}

The mathematical model proposed by Marhl et al. [30] can be simplified [46], by changing the equation (A.31) to:

$$
J_{\text {out }}=k_{\text {out }} C a_{m} \text {, }
$$

where $k_{\text {out }}=0.50625 \mathrm{~s}^{-1}$.

\section{A.4. Model 4}

The mathematical model proposed by Kummer et al. [31] is described by the following differential equations:

$$
\begin{aligned}
& \frac{\mathrm{d} C a_{\mathrm{cyt}}}{\mathrm{d} t}=k_{10} G_{\alpha}-k_{11} \frac{C a_{\mathrm{cyt}}}{C a_{\mathrm{cyt}}+K_{12}}, \\
& \frac{\mathrm{d} G_{\alpha}}{\mathrm{d} t}=k_{1}+k_{2} G_{\alpha}-k_{3} P L C \frac{G_{\alpha}}{G_{\alpha}+K_{4}}-k_{5} C a_{\mathrm{cyt}} \frac{G_{\alpha}}{G_{\alpha}+K_{6}}, \\
& \frac{\mathrm{dPLC}}{\mathrm{d} t}=k_{7} G_{\alpha}-k_{8} \frac{P L C}{P L C+K_{9}} .
\end{aligned}
$$

Parameters for which all calculations are made if not otherwise stated: $k_{1}=0.212, k_{3}=1.52, k_{5}=4.88, k_{7}=1.24$, $k_{8}=32.24, k_{10}=13.58, k_{11}=153, K_{4}=0.19, K_{6}=1.18, K_{9}=29.09, K_{12}=0.16, k_{2}=1.316-2.999$.

\section{References}

[1] Strumwasser F. Types of information stored in single neurons. Invertebrate nervous systems: Their significance for mammalian neurophysiology. Chicago: The University of Chicago Press; 1967. p. 290-319.

[2] Alving B. Spontaneus activity in isolated somata of Aplysia pacemaker neurons. J Gen Physiol 1968;51:29-45.

[3] Dean PM, Matthews EK. Glucose-induced electrical activity in pancreatic islet cells. J Physiol 1970;210:255-64.

[4] Wong RKS, Prince DA. Afterpotential generation in hippocampal pyramidal cells. J Neurophysiol 1981;45:86-97. 
[5] Deschênes M, Roy JP, Steriade M. Thalamic bursting mechanism: An invariant slow current revealed by membrane hyperpolarization. Brain Res 1982;239:289-93.

[6] Crunelli V, Kelly JS, Leresche N, Pirchio M. The ventral and dorsal lateral geniculate nucleus of the rat: Intracellular recordings in vitro. J Physiol 1987;384:587-601.

[7] Harris-Warrick RM, Flamm RE. Multiple mechanisms of bursting in a conditional bursting neuron. J Neurosci 1987;7:2113-28.

[8] Ashcroft F, Rorsman P. Electrophysiology of the pancreatic $\beta$-cell. Prog Biophys Molec Biol 1989;54:87-143.

[9] Johnson SW, Seutin V, North RA. Burst firing in dopamine neurons induced by N-Methyl-D-Aspartate: Role of electrogenic sodium pump. Science 1992;258:665-7.

[10] Rinzel J. A formal classification of bursting mechanisms in excitable systems. Lecture Notes in Biomathematics No. 71. Berlin: Springer-Verlag; 1987.

[11] Smolen P, Terman D, Rinzel J. Properties of a bursting model with two slow inhibitory variables. SIAM J Appl Math 1993;53:861-92.

[12] Holden L, Erneux T. Slow passage through a Hopf bifurcation: Form oscillatory to steady state solutions. SIAM J Appl Math 1993;53:1045-58.

[13] Holden L, Erneux T. Understanding bursting oscillations as periodic slow passages through bifurcation and limit points. J Math Biol 1993;31:351-65.

[14] Pernarowski M. Fast subsystem bifurcations in a slowly varied Liénard system exhibiting bursting. SIAM J Appl Math 1994;54:814-32.

[15] Bertram R, Butte MJ, Kiemel T, Sherman A. Topological and phenomenological classification of bursting oscillations. Bull Math Biol 1995;57:413-39.

[16] de Vries G. Multiple bifurcations in a polynominal model of bursting oscillations. J Nonlin Sci 1998;8:281-316.

[17] Izhikevich EM. Neural excitability spiking and bursting. Int J Bifurcat Chaos 2000;10:1171-266.

[18] Izhikevich EM. Subcritical elliptic bursting of Bautin type. SIAM J Appl Math 2000;60:503-35.

[19] Hoppensteadt FC, Izhikevich EM. Weakly connected neural networks. New York: Springer-Verlag; 1997. p. All.

[20] Berridge MJ, Bootman MD, Lipp P. Calcium — a life and death signal. Nature 1998;395:645-8.

[21] Dupont G, Swillens S, Clair C, Tordjmann T, Combettes L. Hierarchical organization of calcium signals in hepatocytes: From experiments to models. Biochim Biophys Acta 2000;1498:134-52.

[22] Schuster S, Marhl M, Höfer T. Modelling of simple and complex calcium oscillations. From single-cell responses to intercellular signalling. Eur J Biochem 2002;269:1333-55.

[23] Chay TR. Electrical bursting and luminal calcium oscillation in excitable cell models. Biol Cybern 1996;75:419-31.

[24] Woods NM, Cuthbertson KSR, Cobbold PH. Repetitive transient rises in cytoplasmic free calcium in hormone-stimulated hepatocytes. Nature 1986;319:600-2.

[25] Green AK, Dixon CJ, MacLennan AG, Cobbold PH, Fisher MJ. Adenine dinucleotide-mediated cytosolic free $\mathrm{Ca}^{2+}$ oscillations in single hepatocytes. FEBS Lett 1993;322:197-200.

[26] Green AK, Cobbold PH, Dixon CJ. Elevated intracellular cyclic AMP exerts different modulatory effects on cytosolic free Ca ${ }^{2+}$ oscillations induced by ADP and ATP in single rat hepatocytes. Biochem J 1994;302:949-55.

[27] Marrero I, Sanchez-Bueno A, Cobbold PH, Dixon CJ. Taurolithocholate and taurolithocholate 3-sulfate exert different effects on cytosolic free $\mathrm{Ca}^{2+}$ concentration in rat hepatocytes. Biochem J 1994;300:383-6.

[28] Shen P, Larter R. Chaos in intracellular $\mathrm{Ca}^{2+}$ oscillations in a new model for non-excitable cells. Cell Calcium 1995;17:225-32.

[29] Borghans JAM, Dupont G, Goldbeter A. Complex intracellular calcium oscillations. A theoretical exploration of possible mechanisms. Biophys Chem 1997;66:25-41.

[30] Marhl M, Haberichter T, Brumen M. Complex calcium oscillations and the role of mitochondria and cytosolic proteins. BioSystems 2000;57:75-86.

[31] Kummer U, Olsen LF, Dixon CJ, Green AK, Bornberg-Bauer E, Baier G. Switching from simple to complex oscillations in calcium signalling. Biophys J 2000;79:1188-95.

[32] Larter R, Aguda B, Bush C, Lonis T. Multiple steady states, complex oscillations, and the devil's staircase in the peroxidaseoxidase reaction. J Chem Phys 1987;87:5765-71.

[33] Meyer T, Stryer L. Molecular model for receptor-stimulated calcium spiking. Proc Nat Acad Sci 1988;85:5051-5.

[34] Stryer L, Meyer T. Calcium spiking. Ann Rev Biophys Biophys Chem 1991;20:153-74.

[35] Falcke M, Hudson JL. Impact of mitochondrial $\mathrm{Ca}^{2+}$ cycling on pattern formation and stability. Biophys J 1999;77:37-44.

[36] Jafri MS, Vajda S, Pasik P. A membrane model for cytosolic calcium oscillations: A study using Xenopus oocytes. Biophys J 1992;63:235-46.

[37] Wagner J, Keizer J. Effects of rapid buffers on $\mathrm{Ca}^{2+}$ diffusion and $\mathrm{Ca}^{2+}$ oscillations. Biophys J 1994;67:447-56.

[38] Chay TR, Lee SY, Fan SY. Appearance of phase-locked Wenckebach-like rhythms, devil's staircase and universality in intracellular calcium spikes in non-excitable cell models. J Theor Biol 1995;174:21-44.

[39] Houart G, Dupont G, Goldbeter A. Bursting, chaos and birhythmicity originating from self-modulation of the inositol 1,4,5trisphosphate signal in a model for intracellular $\mathrm{Ca}^{2+}$ oscillations. Bull Math Biol 1999;61:507-30.

[40] Haberichter T, Marhl M, Heinrich R. Birhythmicity, trirhythmicity and chaos in bursting calcium oscillations. Biophys Chem 2001;90:17-30.

[41] Brusch L, Lorenz W, Or-Guil M, Bär M, Kummer U. Fold-Hopf bursting in a model for calcium signal transduction. Z Phys Chem 2002;216:487-97. 
[42] Rinzel J. Bursting oscillations in an excitable membrane model. Lecture Notes in Mathematic no. 1151. Berlin: Springer-Verlag; 1985.

[43] Doedel E, Champneys A, Fairgrieve T, Kuznetsov Y. AUTO97: Continuation and bifurcation software for ordinary differential equations. Montreal: Concordia University; 1997.

[44] Ermentrout GB. XPPAUT: The differential equation solving tool. http://www.math.pitt.edu/ bard/xpp/xpp.html, 1996.

[45] Wolf A, Swift JB, Swinney HL, Vastano JA. Determining Lyapunov exponents from a time series. Physica 1985;D16:285-317.

[46] Grubelnk V, Larsen AZ, Kummer U, Olsen LF, Marhl M. Mitochondria regulate the amplitude of simple and complex calcium oscillations. Biophys Chem 2001;94:59-74.

[47] Nejshtadt A. Asymptotic investigation of the loss of stability by an equilibrium as a pair of eigenvalues slowly cross the imaginary axis. Usp Math Nauk 1985;40:190-1.

[48] Baer SM, Erneux T, Rinzel J. The slow passage through a Hopf bifurcation: Delay, memory effects, and resonances. SIAM J Appl Math 1989;49:55-71.

[49] Strogatz SH. Nonlinear Dynamics and Chaos with Applications to Physics, Biology, Chemistry, and Engineering. Cambridge: Perseus Publishing; 1994. p. 326-30.

[50] Hodgin AL, Huxley AF. A quantitative description of membrane current and application to conduction and excitation in nerve. J Physiol 1952;117:500-44.

[51] Wang XJ. Genesis of bursting oscillations in the Hindmarsh-Rose model and homoclinicity to a chaotic saddle. Physica 1993;D62:263-74.

[52] Shorten PR, Wall DJN. A Hodgin-Huxley model exhibiting bursting oscillations. Bull Math Biol 2000;4:695-715.

[53] Li WH, Llopis J, Whitney M, Zlokarnik G, Tsien RY. Cell-permeant caged InsP $\mathrm{P}_{3}$ ester shows that Ca ${ }^{2+}$ spike frequency can optimize gene expression. Nature 1998;392:936-41.

[54] De Koninck P, Schulman H. Sensitivity of CaM kinase II to the frequency of $\mathrm{Ca}^{2+}$ oscillations. Science 1998;279:227-30.

[55] Dolmetsch RE, $\mathrm{Xu} \mathrm{K}$, Lewis RS. Calcium oscillations increase the efficiency and specificity of gene expression. Nature 1998;392:933-6.

[56] Marhl M, Schuster S, Brumen M. Mitochondria as an important factor in the maintenance of constant amplitudes of cytosolic calcium oscillations. Biophys Chem 1998;71:125-32.

[57] Bayer KU, De Koninck P, Schulman H. Alternative splicing modulates the frequency-dependent response of CaMKII to Ca ${ }^{2+}$ oscillations. EMBO J 2002;21:3590-7.

[58] Hudmon A, Schulman H. Structure-function of the multifunctional $\mathrm{Ca}^{2+} /$ calmodulin-dependent protein kinase II. Biochem J 2002;364:593-611.

[59] Prank K, Gabbiani F, Brabant G. Coding efficiency and information rates in transmembrane signalling. Biosystems 2000;55:1522.

[60] Prank K, Kropp M, Brabant G. Humoral coding and decoding. Complexity Biol Inform Processing 2001;239:96-110.

[61] Izhikevich EM. Synchronization of elliptic bursters. SIAM Rev 2001;43:315-44.

[62] Poon C-S, Merrill CK. Decrease of cardiac chaos in congestive heart failure. Nature 1997;389:492-5.

[63] Goldbeter A. Biochemical oscillations and cellular rhythms. Cambridge: Cambridge University Press; 1996. p. 89-160 and 349406.

[64] Galvanovskis J, Sandblom J. Periodic forcing of intracellular calcium oscillators. Theoretical studies of the effects of low frequency fields on the magnitude of oscillations. Bioelectrochem Bioenerg 1998;46:161-74.

[65] Sandblom J, Galvanovskis J. Electromagnetic field absorption in stochastic cellular systems: enhanced signal detection in ion channels and calcium oscillators. Chaos, Solitons \& Fractals 2000;11:1905-11.

[66] Perc M, Marhl M. Sensitivity and flexibility of regular and chaotic calcium oscillations. Biophys Chem 2003, in press. 